\title{
Evolución de operaciones por enfermedades del intestino proximal y glándulas anexas realizadas en Chile entre 2011 y 2016
}

\author{
Attila Csendes J. ${ }^{1}$, Nicole Cuneo B. ${ }^{1}$, Manuel Figueroa-Giralt ${ }^{1}$ y Omar Orellana E. ${ }^{1}$
}

\section{Evolution of surgeries due to diseases of the proximal intestine and anexal glands perfomed in Chile between 2011 and 2016}

Introduction: The number of operations performed in Chile due to diseases for the foregut, have been every time more precise and complete in the National Register Data of Statistics in Health (MINSAL), and published in several reports (years 1983, 2005 and 2011). Objectives: a) to incorporate data of new operations not included before and b) to compare the results of high complex operations reported on 2011 and 2016. Material and Methods: from the DEIS (Department of Statistics and Information of Health) we obtained the total number of operations performed during 2016, and the operative mortality at the time of discharge from the hospital for different diseases of the foregut been the majority malignant tumors: esophageal cancer, gastric cancer, pancreatic cancer, liver and bile ducts carcinoma, and cancer of the gallbladder. The results were compared to dose obtained the year 2011. Results: The annual lethality of digestive malignant tumors showed an increase in pancreatic and liver cancer, while a decrease was observed in patients with esophageal cancer al gallbladder cancer. Operations for benign pathology like esophagomiotomy and laparoscopic antireflux surgery showed no mortality, as well as gastric bypass. Laparoscopic cholecistectomy, and laparoscopic sleeve gastrectomy presented a very low mortality. Surgical procedures for malignant diseases showed a decrease in operative mortality compare to 2011, although not significant. Conclusions: The present study shows an increase in the operations for foregut diseases performed during 2016 compared with the year 2011, as well as a decrease in operative mortality.

Key words: tumors of foregut; morbid obesity; achalasia; gastroesophageal reflux disease; laparoscopic cholecystectomy.

\section{Resumen}

Introducción: Los datos sobre número de operaciones realizadas en Chile en patología del intestino proximal y glándulas anexas, han sido cada vez más precisas en los registros estadísticos del MINSAL en los últimos años. Objetivo: a) Incorporar datos de otras operaciones no incluidas en reportes previos, b) comparar los resultados de operaciones de alta complejidad reportadas el año 2011 y confrontarlos con los datos del año 2016. Material y Método: En el DEIS (Departamento de Estadística e Información de Salud) se obtuvo el número total de operaciones realizadas en Chile el año 2016 y mortalidad operatoria al momento del egreso de diferentes patologías del intestino proximal y glándulas anexas, siendo la mayoría tumores malignos: cáncer de esófago, estómago, páncreas, hígado y vías biliares intrahepáticas y vesícula biliar. Los resultados se compararon con los obtenidos del año 2011. Resultados: La letalidad anual de cánceres digestivos demostró un alza del cáncer de páncreas e hígado, mientras que se aprecia una baja en el cáncer de esófago y vesícula biliar. Operaciones de patología benigna, como la esofagocardiomiotomía y cirugías antirreflujo laparoscópica no mostraron mortalidad, al igual que el bypass gástrico. La colecistectomía y la gastrectomía vertical laparoscópicas, tuvieron una muy baja mortalidad operatoria. En las intervenciones quirúrgicas por tumores malignos se apreció en todos una disminución de la mortalidad operatoria comparado con el año 2011, aunque no fue estadísticamente significativo. Conclusión: El presente estudio muestra un aumento de las operaciones por enfermedades del intestino proximal y glándulas anexas así como una disminución de la mortalidad operatoria el año 2016 comparado con el 2011.

Palabras clave: tumores del intestino proximal; obesidad mórbida; acalasia; reflujo gastroesofágico; colecistectomía laparoscópica.
'Departamento de Cirugía,

Hospital Clínico de la Universidad de Chile. Santiago, Chile.

Recibido el 23 de abril de 2018 y aceptado para publicación el 11 de julio de 2018

Correspondencia a: Dr. Attila Csendes J. acsendes@hcuch.cl 


\section{Introducción}

La patología digestiva representa la cirugía más frecuentemente realizada en Chile, después de las cesáreas. En publicaciones previas informamos el número de operaciones por patología gastrointestinal alta realizadas en Chile durante los años $1982^{1}$, 2004-2005 y $2011^{3}$. Los datos aportados por la Oficina de Estadística de Salud (DEIS) del Ministerio de Salud fueron progresivamente más completos, ya que inicialmente sólo se incluyeron los datos de las operaciones realizadas en los Hospitales del Ministerio de Salud. Posteriormente se incluyeron los datos del sistema privado de salud, aportando un panorama bastante fiel de la situación nacional referente al número de intervenciones realizadas y su mortalidad operatoria.

Los objetivos del presente estudio fueron responder: a) ¿Cuál es la prevalencia de operaciones de patologías benignas del esófago que no fueron incluidas en reportes previos? y b) ¿Cuáles son los cambios de la frecuencia y mortalidad operatoria de operaciones oncológicas de alta complejidad realizadas el año 2016, comparando esta información con los datos del año 2011 ?

\section{Material y Método}

Estudio ecológico descriptivo y analítico transversal, realizado con el apoyo y ayuda del Departamento de Estadística e Información de la Salud (DEIS) de la División de Planificación Sanitaria (DIPLAS) del Ministerio de Salud. Se determinó el número total de intervenciones quirúrgicas realizadas en Chile durante el año 2016. Esta información se generó a partir del Informe Estadístico de Egreso Hospitalario. Por norma, los diagnósticos de egreso deben ser codificados por codificadores de morbilidad, que aplican reglas de codificación establecidos en la Clasificación Estadística Internacional de Enfermedades y Problemas relacionados con la Salud, que están vigentes en el país, designados como CIE-10. Estos procedimientos estadísticos permiten agrupar causas, que para nuestro interés son principalmente los tumores malignos, cuyos códigos son:

- Tumor maligno de esófago: C15.

- Tumor maligno de estómago: C16.

- Tumor maligno de páncreas: C25.

- Tumor maligno de hígado y vías biliares intrahepáticas: C22.

- Tumor maligno de vesícula biliar: C10 y C23.
En el caso de las operaciones realizadas se empleó la codificación de FONASA que es universalmente empleada en Chile:

\section{1) Patología esofágica}

a. Esofagectomía por cáncer 17-04-056 y 17-04-057.

b. Esofagocardiomiotomía por acalasia 17-04-054.

c. Fundoplicatura laparoscópica 18-02-024.

d. Fundoplicatura y hernioplastía hiatal 18-02-024 junto con 18-02-001.

\section{2) Cirugía por cáncer gástrico}

a. Gastroyeyunoanastomosis 18-02-012

b. Gastrectomía subtotal con disección ganglionar 18-02-017

c. Gastrectomía total o ampliada $18-02-022$ y $18-02-023$

\section{3) Cirugía de la obesidad}

a. Bypass laparoscópico 18-02-012

b. Gastrectomía vertical laparoscópica 18-02-018

\section{4) Cirugía por tumores pancreáticos}

a. Pancreatectomía parcial o distal. 18-02-045

b. Pancreatoduodenectomía 18-02-047

\section{5) Cirugía por colecistolitiasis}

a. Colecistectomía laparoscópica 18-02-081

b. Colecistectomía laparotómica 18-02-028

\section{6) Cirugía por tumores malignos de hígado}

a. Hepatectomía segmentaria 18-02-039

b. Lobectomía hepática 18-02-041

\section{7) Cirugía por cáncer de vesícula biliar}

a. Colecistectomía 18-02-028

b. Colédocoentero anastomosis 18-02-032

c. Segmentectomía hepática 18-02-039 


\section{Estadística}

Las variables categóricas se expresaron con porcentaje, mientras que las continuas se expresaron en promedio y desviación estándar (DS) o mediana y rango intercuartil en caso de ser una variable paramétrica o no respectivamente.

Se utilizó test de $\chi^{2}, t$ de Student y regresión de Poissont según característica y distribución de las variables. Para el análisis estadístico analítico se empleó el programa STATA 13, se consideró estadísticamente significativo un $\mathrm{p}<0,05$. Además, se empleó el Incidence Rate Ratio (IRR) que representa el cambio anual de la tasa de incidencia de un período a otro.

\section{Definiciones}

Mortalidad quirúrgica: Tasa de pacientes fallecidos entre la cirugía y los 30 días posoperatorios.

La tasa de mortalidad por patología específica se ajustó a 100.000 habitantes.

\section{Resultados}

La Tabla 1 señala la letalidad anual a nivel nacional de cánceres del intestino proximal y de glándulas anexas, comparando los datos de 3 años diferentes: 2005, 2011 y 2016. Se aprecia que el cáncer de esófago tiene una clara tendencia a la disminución en comparar el año 2005 con el año 2016. El cáncer de estómago permanece estable en el período de análisis, mientras que el cáncer de páncreas presenta un aumento en la tasa por 100.000 habitantes de un 39\%. Por su parte el tumor maligno de hígado muestra un alza tanto en el número absoluto de pacientes cómo en su tasa. El cáncer de la vesícula biliar presenta una diminución en la tasa de letalidad en forma progresiva. Sin embargo, ninguno de los cambios en las tasas muestra una significación estadística. Por otra parte los cambios se pueden analizar con el IRR, demostrando una disminución anual de 4\%,1\% y 2\% para los cánceres de esófago, estómago vesícula respectivamente, mientras que para el cáncer de páncreas y cáncer hepático se aprecia un alza anual de 3\% y $6 \%$ respectivamente.

En la Tabla 2, se muestran las operaciones realizadas por patología esofágica benigna y maligna durante el año 2016. Las intervenciones por patología benigna no registran mortalidad, mientras que la esofagectomía por cáncer presentó una mortalidad de $5 \%$.

Las operaciones relacionadas con cáncer gástrico se observan en la Tabla 3. La gastroyeyunoanastomosis como operación paliativa se realizó en el $3,7 \%$ de los pacientes operados, con una mortalidad elevada. La gastrectomía total se realizó en el 72,7\% de los casos con una mortalidad del $2 \%$ y la gastrectomía subtotal en el $23,5 \%$ con una mortalidad del $1,2 \%$. Ambas operaciones aumentaron en un $41 \%$ y $35 \%$ respectivamente, comparadas con el año 2011 , pero sin significación estadística.

Tabla 1. Letalidad anual del cáncer digestivo del intestino proximal y glándulas anexas

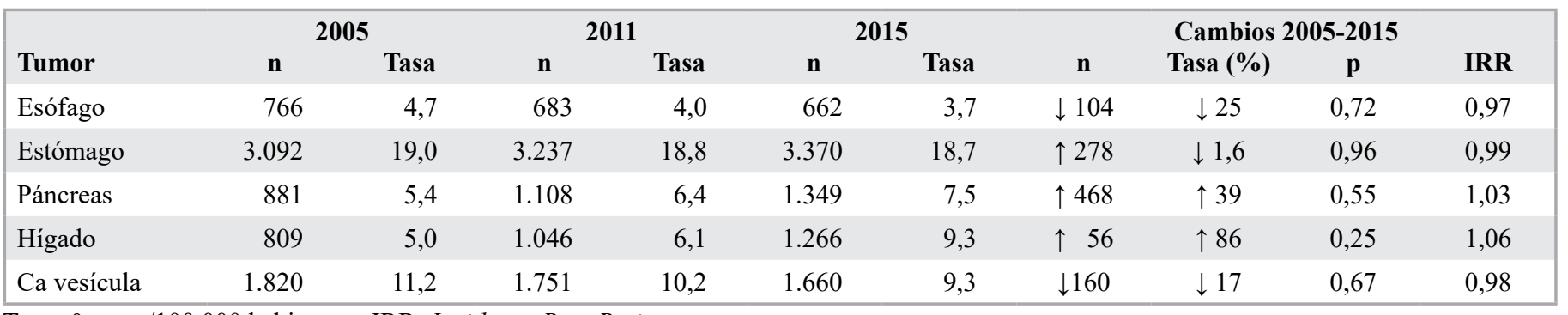

Tasa ${ }^{\circ}$ casos/100.000 habitantes; IRR: Incidence Rate Ratio.

Tabla 2. Número de operaciones relacionadas con patología esofágica $(n=488)$ en Chile, año 2016

\begin{tabular}{|lccc|}
\hline Tipo de operación & n & Mortalidad & Cambio respecto al año 2011 \\
Esofagocardiomiotomía por acalasia & 76 & 0 & $i ?$ \\
Cirugía antirreflujo laparoscópica & 256 & 0 & $i ?$ \\
Cirugía antirreflujo y hernioplastía hiatal laparoscópica & 115 & 0 & $i ?$ \\
Esofagectomía por cáncer & 41 & $2(4,9 \%)$ & $=$ \\
\hline
\end{tabular}


Las operaciones realizadas por obesidad en el país el año 2016 fueron en total 5.252 (Tabla 4). De ellos, el bypass gástrico se efectuó en el 29,5\% de los pacientes sin mortalidad. La gastrectomía vertical o "manga" que se realizó en el 70,5\%, con una mortalidad del $0,13 \%$. Ambas operaciones tuvieron un incremento de $85 \%$ a $87 \%$ comparados con el año 2011, sin significación estadística.

La colecistectomía por colecistolitiasis se muestra en la Tabla 5. La colecistectomía laparoscópica se realizó en el 95\% de los pacientes con una muy baja mortalidad operatoria, cifra 20 veces menor que la mortalidad por colecistectomía laparotómica. El aumento de la colecistectomía laparoscópica y la disminución de vía laparotómica fueron estadísticamente significativos.
La Tabla 6 señala las operaciones realizadas para tumores pancreáticos, destacando que la pancreatectomía parcial correspondió al 29\% de los pacientes y no presentó mortalidad operatoria, mientras la pancreatoduodenectomía mostró una mortalidad del $7,1 \%$. Ambas intervenciones aumentaron en $123 \%$ y $50 \%$ respectivamente, sin significación estadística.

La Tabla 7 detalla el número de operaciones y mortalidad por tumores malignos del hígado. Tanto la hepatectomía segmentaria como la lobectomía hepática se realizaron en proporciones similares $(49 \%$ y $51 \%$ respectivamente), con una baja mortalidad operatoria.

La Tabla 8 muestra las operaciones realizadas por carcinoma de la vesícula biliar que pueden ser diferenciadas exclusivamente por la codificación

Tabla 3. Número de operaciones realizadas por cáncer gástrico $(n=1.420)$ en Chile, año 2016

\begin{tabular}{|lccccc|}
\hline Tipo de operación & n 2011 & n 2016 & Cambio respecto al año 2011 & p & IRR \\
Gastroyeyunoanastomosis & & 53 & $i ?$ & - & - \\
Gastrectomía subtotal & 237 & 334 & $\uparrow 41 \%$ & 0,9 & 1,42 \\
Gastrectomía total & 795 & 1.035 & $\uparrow 35 \%$ & 0,9 & 0,88 \\
\hline
\end{tabular}

IRR: Incidence rate ratio.

Tabla 4. Número de operaciones realizadas por obesidad $(n=5.252)$ en Chile, año 2016

\begin{tabular}{|lrcccc|}
\hline Tipo de operación & $\mathbf{n ~ 2 0 1 1}$ & $\mathbf{n ~ 2 0 1 6}$ & Cambio respecto al año 2011 & p & IRR \\
\hline Bypass gástrico laparoscópico & 828 & 1.550 & $\uparrow 87 \%$ & 0,24 & 1,13 \\
Gastrectomía vertical laparoscópica & 2.006 & 3.702 & $\uparrow 85 \%$ & 0,11 & 1,11 \\
\hline
\end{tabular}

IRR: Incidence rate ratio.

Tabla 5. Número de operaciones realizadas por colecistolitiasis $(n=59.420)$ en Chile, año 2016

\begin{tabular}{|lrrcccc|}
\hline Tipo de operación & n 2011 & n 2016 & Cambio respecto al año 2011 & P & IRR \\
\hline Colecistectomía laparoscópica & 42.483 & 56.523 & $\uparrow 33 \%$ & 0,0006 & 1,04 \\
\hline Colecistectomía laparotómica & 7.669 & 2.897 & $\downarrow 62 \%$ & 0,0002 & 0,81 \\
\hline
\end{tabular}

IRR: Incidence rate ratio.

Tabla 6. Número de operaciones realizadas por patología pancreática $(n=198)$ en Chile, año 2016

\begin{tabular}{|lccccc|}
\hline Tipo de operación & n 2011 & n 2016 & Cambio respecto al año 2011 & p & IRR \\
\hline Pancreatectomía parcial/distal & 26 & 58 & $\uparrow 123 \%$ & 0,8 & 1,16 \\
\hline Pancreatoduodenectomía & 76 & 140 & $\uparrow 50 \%$ & 0,7 & 1,12 \\
\hline
\end{tabular}

IRR: Incidence rate ratio. 
Tabla 7. Número de operaciones realizadas por tumores malignos del hígado $(\mathrm{n}=152)$, año 2016

\begin{tabular}{|lccc|}
\hline Tipo de operación & n & Mortalidad & Cambio respecto al año 2011 \\
\hline Hepatectomía segmentaria & 69 & $2(2,9 \%)$ & $¿ ?$ \\
Lobectomía hepática & 83 & $3(3,6 \%)$ & $i ?$ \\
\hline
\end{tabular}

empleada. La segmentectomía hepática fue la intervención más utilizada en el $80 \%$ de los casos. No se cuenta con datos diferenciales de hepatectomías mayores o derivaciones biliodigestivas.

En la Tabla 9 se resume la mortalidad operatoria de 5 operaciones de alta complejidad realizadas por tumores malignos y su cambio respecto al año 2011. En todas las intervenciones se aprecia una disminución de esta mortalidad, sin significación estadística.

\section{Discusión}

Los resultados del presente estudio comparados con los datos publicados con anterioridad ${ }^{2,3}$, demuestran que: a) la colecistectomía es la intervención quirúrgica abdominal más frecuente, b) hay un aumento de las operaciones por cáncer digestivo proximal, así como de las glándulas anexas, c) la mortalidad operatoria de estas intervenciones ha disminuido considerablemente en todo el país y d) los datos entregados por el MINSAL son cada vez más completos y precisos, aunque aún insuficientes.

En el presente análisis hemos incluido por primera vez en Chile, algunas operaciones por enfermedades benignas de esófago, de las cuales sólo existen publicaciones de centros quirúrgicos específicos, pero sin mención a la realidad nacional total. Es el caso de la esofagocardiomiotomía por acalasia y la fundoplicatura laparoscópica con o sin hernioplastía hiatal, en las cuales tenemos varios reportes completos previos ${ }^{4,5}$. En este estudio, sin embargo, no fue posible precisar en cuántos pacientes con hernia hiatal se colocó una malla de refuerzo adicional, lo que debería establecerse en el futuro. Destaca el hecho que no se reporta mortalidad operatoria en estos procedimientos, lo que había sido publicado por nosotros previamente ${ }^{4,5}$.

La otra novedad del presente estudio fue incorporar los datos sobre cirugía de tumores malignos del hígado y vías biliares intrahepáticas. Estos corresponden a hepatocarcinoma, metástasis hepática y colangiocarcinoma. Llama la atención la proporción similar de segmentectomía y lobectomía hepática, en cuanto a que todas las publicaciones internacionales señalan que se realizan más segmentectomías
Tabla 8. Número de operaciones realizadas por cáncer de la vesícula biliar $(n=141)$ año 2016

\begin{tabular}{|lc|}
\hline Tipo de operación & n \\
\hline Colecistectomía simple & $24(17 \%)$ \\
Colédocoentero anastomosis & $4(3 \%)$ \\
\hline Hepatectomía segmentaria & $113(80 \%)$ \\
\hline
\end{tabular}

Tabla 9. Mortalidad operatoria del año 2011 y el año 2016 de intervenciones de alta complejidad por cáncer digestivo alto en Chile

\begin{tabular}{|lccccc|}
\hline Tipo de operación & \multicolumn{2}{c}{ Mortalidad } & Cambio & & \\
& $\mathbf{2 0 1 1}$ & $\mathbf{2 0 1 6}$ & $\mathbf{2 0 1 1 - 2 0 1 6}$ & P & IRR \\
\hline Esofagectomía & $9,5 \%$ & $4,9 \%$ & $\downarrow 48 \%$ & 0,9 & 0,95 \\
Gastrectomía subtotal & $2,9 \%$ & $1,2 \%$ & $\downarrow 58 \%$ & 0,9 & 0,89 \\
Gastrectomía total & $3,4 \%$ & $2,0 \%$ & $\downarrow 47 \%$ & 0,9 & 0,87 \\
Pancreatectomía parcial & 0 & 0 & $i ?$ & - & - \\
Pancreatoduodenectomía & $8,7 \%$ & $7,1 \%$ & $\downarrow 18 \%$ & 0,94 & 0,88 \\
\hline
\end{tabular}

IRR: Incidence rate ratio.

que lobectomías hepáticas en cualquier centro ${ }^{6,7}$. Esto puede ser una debilidad en la información precisa que se coloca en la hoja de estadística. Como ejemplo, el DEIS nos reportó un total de 149 lobectomías hepáticas realizadas el año 2016, de las cuales sólo el 72 (48,3\%) eran por tumores malignos y 77 por una gran cantidad de diferentes diagnósticos no relacionados con malignidad, siendo un total de 27 diagnósticos, que deberán de ser muy aclarados en el futuro. Lo mismo se aplica a la búsqueda de las operaciones para cáncer de vesícula biliar: como no hay un código preciso para la operación de elección (segmentectomía IVb-V más linfadenectomía), la codificación que se emplea en la práctica es aquella que se asemeje a lo realizado.

La situación del cáncer gástrico en Chile ha sido publicada recientemente por nosotros ${ }^{8}$. A pesar que las tasas de mortalidad se mantienen sin cambios, llamó la atención un aumento de las operaciones y una baja en la mortalidad operatoria. Este hecho se puede deber en gran parte al establecimiento del 
plan AUGE para esta patología, que permite un acceso más rápido y oportuno para la intervención quirúrgica. Las bajas cifras de mortalidad operatoria similares a los existentes en Japón y Corea reflejan un progreso en el diagnóstico precoz y en el manejo multidisciplinario de los pacientes, así como la creación de equipos quirúrgicos expertos en esta enfermedad en todo Chile, la mayor tasa de mortalidad evidenciada en cirugía paliativa (Tabla 3) puede explicarse por el estado oncológico avanzado de la enfermedad y el perfomance status del paciente, aunque esta suposición no puede desprenderse de nuestros resultados ya que estos detalles no se especifican en la hoja de egreso hospitalario.

Las operaciones por obesidad ocupan un lugar preponderante comparado con el año 2011, observando un aumento en ambas intervenciones. Esto puede deberse a un mejor registro de esta patología, que en su inmensa mayoría se realiza en centros privados. El hecho que la gastrectomía vertical o "manga" se realiza en más del $70 \%$ de los casos, nos preocupa dada la alta incidencia de reflujo gastroesofágico que se produce después del procedimiento 9 . En nuestro centro, el bypass gástrico se realiza en $60 \%$ y la gastrectomía vertical en $40 \%$. Además, esta intervención presentó mortalidad operatoria, lo que debe ser evaluado por el cirujano tratante al momento de realizar el consentimiento informado.

La colecistectomía por colecistolitiasis sigue siendo la intervención quirúrgica más frecuente en cualquier servicio de cirugía del país, por lo menos desde 1983 cuando la informamos por primera vez en Chile ${ }^{1}$. Las cifras actuales muestran un significativo incremento respecto al año 2011 y por primera vez podemos conocer la mortalidad operatoria global que es un logro para la cirugía laparoscópica que se realiza en el $95 \%$ de los pacientes. La cifra de mortalidad mayor para la colecistectomía laparotómica es posible dado a que esta vía de abordaje se emplea en pacientes de mayor complejidad, habitualmente con colecistitis aguda en etapas avanzadas, con abscesos perivesiculares, perforación o necrosis de vesícula, síndrome de Mirizzi II, III y $\mathrm{IV}^{10}$, en los que muchas veces se comienza por vía laparoscópica y que debe convertirse a vía laparotómica por todas las dificultades técnicas.

Con respecto a la cirugía pancreática por tumores, es imposible precisar si son benignos o malignos. Sin embargo, sabemos que la inmensa mayoría de las pancreatoduodenectomías se realiza por cáncer de la cabeza de páncreas o proceso uncinado ${ }^{11}$. En cambio, en la pancreatectomía distal o parcial, en que no se aprecie mortalidad operatoria, con fre- cuencia se plantea por tumores benignos del cuerpo o cola de páncreas, como quistes serosos, IPMN de conductos secundarios $\mathrm{u}$ otros $-\mathrm{y}$ se realiza por vía laparoscópica en la mayoría- lo que es el gold standard actual.

Cuando se analiza la mortalidad operatoria de las intervenciones por cáncer digestivo comparando el año 2011 con el año 2016, se puede observar una disminución no significativa. Estos cambios pudiesen estar en el contexto académico-científico de la Sociedad de Cirujanos de Chile, la cual ha creado departamentos específicos de cirujanos esófagogástricos y hepato-bilio-pancreáticos, los cuales poseen Jornadas Científicas anuales para la difusión del conocimiento y estándares de tratamiento.

Una limitación del presente estudio radica en la codificación del DEIS, que sólo detalla el estado de egreso hospitalario como vivo o fallecido, sin especificar la mortalidad operatoria a los 30 y 90 días posoperatoria como debe ser en la actualidad. Este hecho es otra debilidad del sistema de registro que debiera mejorarse.

En conclusión, el presente estudio muestra un aumento de las intervenciones quirúrgicas del intestino proximal y de las glándulas anexas comparadas con el año 2011; así como una disminución de la mortalidad operatoria. Llamamos a todos los cirujanos de Chile a llenar con mucha precisión la hoja de estadística al egreso del paciente, con el objetivo de lograr una recolección de datos más precisos en el futuro próximo.

\section{Agradecimientos}

Los autores agradecen muy sinceramente la gran colaboración de la Dra. Verónica Rojas, Jefa del Departamento DEIS y a la Sra. Alejandra Gutiérrez, Ingeniero Estadístico, ambas de la División de Planificación Sanitaria del Ministerio de Salud. También agradecen la ayuda de la Dra. Claudia Albornoz y Dra. Natalia Moreno, expertas en estadística.

\section{Responsabilidades éticas}

Protección de personas y animales. Los autores declaran que para esta investigación no se han realizado experimentos en seres humanos ni en animales.

Confidencialidad de los datos. Los autores declaran que en este artículo no aparecen datos de pacientes.

Conflictos de interés: no hay. 


\section{Referencias}

1. Csendes A, Medina E, Medina AM. Características de los Servicios de Cirugía en Chile y operaciones infrecuentes realizadas en diversos tipos de hospitales. Rev Med Chile 1983;111:1065-74.

2. Csendes A, González G. Cirugía digestiva más frecuente en Chile, excluyendo colorrectal. Rev Chil Cir. 2008;60:379-86.

3. Csendes A. Número total de operaciones de patología digestiva alta en Chile año 2011. Rev Chil Cir. 2015;67:61-4.

4. Csendes A, Braghetto I, Burdiles P. Tratamiento quirúrgico de la acalasia esofágica. Experiencia en 328 pacientes. Rev Chil Cir. 2012;64:46-51.
5. Csendes A, Díaz JC, Burdiles P, Maluenda P. Detalles técnicas y morbimortalidad de la fundoplicatura de Nissen laparoscópica en 503 pacientes con reflujo gastroesofágico. Rev Chil Cir. 2016;68:143-9.

6. Machairas N, Papaconstantinou D, Stamopoulos P, Prodromidou A, Garoufalia Z, Spartalis E, et al. The Emerging Role of Laparoscopic Liver Resection in the Treatment of Recurrent Hepatocellular Carcinoma: A Systematic Review. Anticancer Res. 2018;38:3181-6.

7. Wicherts DA, de Haas RJ, Salloum C, Andreani P, Pascal G, Sotirov D, et al. Repeat hepatectomy for recurrent colorectal metastases. Br J Surg.
2013;100:808-18.

8. Csendes A, Figueroa M. Situación del cáncer gástrico en el mundo y Chile. Rev Chil Cir. 2017;69:502-7.

9. Csendes A, Braghetto I. Changes in the Anatomy and Physiology of the Distal Esophagus and Stomach after Sleeve Gastrectomy. Obes Weight Loss Ther. 2016;6:1-9.

10. Csendes A., Díaz JC. Burdiles P, Maluenda P. Mirizzi syndrome and cholecistolithiasis fistula: a unifying classification. Br J Surg. 1989;76:1139-43.

11. Cameron J, Riall T, Coleman J, Belcher K. One Thousand Consecutive Pancreaticoduodenectomies. Ann Surg. 2006;244:10-5 